\title{
PA-043 MALARIA PREVENTION PRACTICES AMONG PREGNANT MOTHERS IN OSOGBO, NIGERIA
}

Adelani Tijani. Bayero University Kano, Nigeria

10.1136/bmjgh-2016-000260.77

Background Pregnant women are susceptible to symptomatic malaria due to invasion of the placenta by Plasmodium. The study aimed to find out the preventive measures put in place by pregnant mothers against malaria.

Methods It is a descriptive cross-sectional survey comprising 294 pregnant women selected randomly in three hospitals in Osogbo. The instrument used for data collection was a selfdeveloped, structured questionnaire with reliability of 0.802 using Cronbach's alpha coefficient.

Results The results show that $87.8 \%$ of the respondents had adequate knowledge about malaria in pregnancy and $75.5 \%$ of them were knowledgeable about various available measures in malaria prevention. However, only $34.4 \%$ used the insecticide treated nets (ITNs) and $21.4 \%$ used intermittent preventive therapy (IPTp). Findings also revealed that the respondents practiced other preventive measures such as clearing of surrounding bushes (12.8\%), maintenance of drainages and netting of windows and doors (15.4\%). The results of the study revealed that various barriers to the use of ITNs were deficient knowhow (45.9\%), spousal disapproval (36.7\%), socio-cultural misconceptions about sleeping under ITNs (18.8\%) and unaffordability of ITNs (45.5\%). The hypotheses were tested using Pearson's chi-square method at 0.05 level of significance. There is no significant relationship between the pregnant mothers' knowledge and their practice of malaria prevention. However, there are respective significant relationships between the age, parity and educational status and practice of malaria prevention. Conclusions It was concluded that the practice of malaria prevention was generally low among the respondents. It was therefore recommended that concerted effort be put in place by the nurses, more especially public health nurses to address the barriers to utilisation of the universally accepted effective methods of malaria prevention. This could be done through mass health education to market women at regular interval. 